\title{
SECOND SEASON QUIET OBSERVATIONS: MEASUREMENTS OF THE COSMIC MICROWAVE BACKGROUND POLARIZATION POWER SPECTRUM AT $95 \mathrm{GHz}$
}

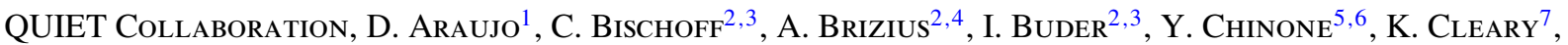 \\ R. N. Dumoulin ${ }^{1}$, A. Kusaka ${ }^{2,8}$, R. Monsalve ${ }^{9,10}$, S. K. Næess ${ }^{11}$, L. B. Newburgh ${ }^{1,8}$, R. Reeves ${ }^{7}$, I. K. Wehus ${ }^{12,13}$,

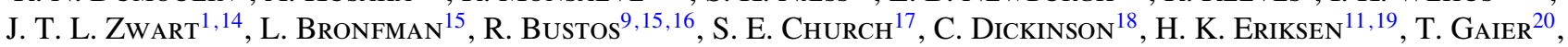 \\ J. O. Gundersen ${ }^{9}$, M. Hasegawa ${ }^{5}$, M. Hazumi ${ }^{5}$, K. M. Huffenderger ${ }^{9}$, K. Ishidoshiro ${ }^{5}$, M. E. Jones ${ }^{12}$, P. Kangaslahti $^{20}$,

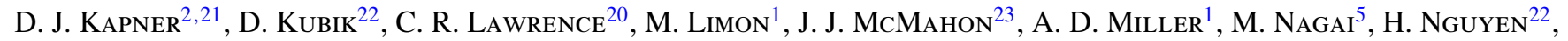 \\ G. Nixon ${ }^{8,24}$, T. J. Pearson ${ }^{7}$, L. Piccirillo ${ }^{18}$, S. J. E. RadFord ${ }^{7}$, A. C. S. Readhead ${ }^{7}$, J. L. Richards ${ }^{7}$, D. Samtleben ${ }^{4,25}$,

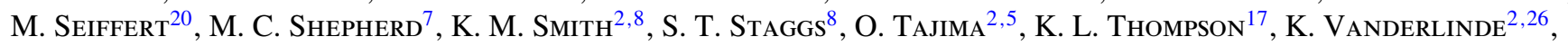 \\ AND R. WILliamSON ${ }^{1,2}$ \\ ${ }^{1}$ Department of Physics and Columbia Astrophysics Laboratory, Columbia University, New York, NY 10027, USA \\ ${ }^{2}$ Kavli Institute for Cosmological Physics, Department of Physics, Enrico Fermi Institute, \\ The University of Chicago, Chicago, IL 60637, USA; ibuder@uchicago.edu \\ ${ }^{3}$ Harvard-Smithsonian Center for Astrophysics, MS 43, Cambridge, MA 02138, USA \\ ${ }^{4}$ Max-Planck-Institut für Radioastronomie, Auf dem Hügel 69, D-53121 Bonn, Germany \\ ${ }^{5}$ High Energy Accelerator Research Organization (KEK), 1-1 Oho, Tsukuba, Ibaraki 305-0801, Japan \\ ${ }^{6}$ Astronomical Institute, Graduate School of Science, Tohoku University, Aramaki, Aoba, Sendai 980-8578, Japan \\ ${ }^{7}$ Cahill Center for Astronomy and Astrophysics, California Institute of Technology, 1200 E. California Blvd M/C 249-17, Pasadena, CA 91125, USA \\ ${ }^{8}$ Joseph Henry Laboratories of Physics, Jadwin Hall, Princeton University, Princeton, NJ 08544, USA \\ ${ }^{9}$ Department of Physics, University of Miami, 1320 Campo Sano Drive, Coral Gables, FL 33146, USA \\ ${ }^{10}$ School of Earth and Space Exploration, Arizona State University, 781 E. Terrace Road, Tempe, AZ 85287, USA \\ ${ }^{11}$ Institute of Theoretical Astrophysics, University of Oslo, P.O. Box 1029 Blindern, NO-0315 Oslo, Norway \\ 12 Department of Astrophysics, University of Oxford, Keble Road, Oxford OX1 3RH, UK \\ ${ }^{13}$ Department of Physics, University of Oslo, P.O. Box 1048 Blindern, NO-0316 Oslo, Norway \\ ${ }^{14}$ Physics Department, University of the Western Cape, Private Bag X17, Bellville 7535, South Africa \\ ${ }^{15}$ Departamento de Astronomía, Universidad de Chile, Casilla 36-D, Santiago, Chile \\ ${ }^{16}$ Departamento de Astronomía, Universidad de Concepción, Casilla 160-C, Concepción, Chile \\ ${ }^{17}$ Kavli Institute for Particle Astrophysics and Cosmology and Department of Physics, Stanford University, \\ Varian Physics Building, 382 Via Pueblo Mall, Stanford, CA 94305, USA \\ 18 Jodrell Bank Centre for Astrophysics, Alan Turing Building, School of Physics and Astronomy, \\ The University of Manchester, Oxford Road, Manchester M13 9PL, UK \\ ${ }^{19}$ Centre of Mathematics for Applications, University of Oslo, P.O. Box 1053 Blindern, NO-0316 Oslo, Norway \\ 20 Jet Propulsion Laboratory, California Institute of Technology, 4800 Oak Grove Drive, Pasadena, CA 91109, USA \\ ${ }^{21}$ Micro Encoder Inc., Kirkland, WA 98034, USA \\ ${ }^{22}$ Fermi National Accelerator Laboratory, Batavia, IL 60510, USA \\ ${ }^{23}$ Department of Physics, University of Michigan, 450 Church Street, Ann Arbor, MI 48109, USA \\ ${ }^{24}$ Tradeworx, Inc., 10 Broad Street, Third Floor, Red Bank, NJ 07701, USA \\ ${ }^{25}$ Nikhef, Science Park, Amsterdam, The Netherlands \\ ${ }^{26}$ Department of Physics, McGill University, 3600 Rue University, Montreal, Quebec H3A 2T8, Canada \\ Received 2012 July 20; accepted 2012 September 23; published 2012 November 16
}

\begin{abstract}
The Q/U Imaging ExperimenT (QUIET) has observed the cosmic microwave background (CMB) at 43 and $95 \mathrm{GHz}$. The $43 \mathrm{GHz}$ results have been published in a previous paper, and here we report the measurement of CMB polarization power spectra using the $95 \mathrm{GHz}$ data. This data set comprises $5337 \mathrm{hr}$ of observations recorded by an array of 84 polarized coherent receivers with a total array sensitivity of $87 \mu \mathrm{K} \sqrt{\mathrm{s}}$. Four low-foreground fields were observed, covering a total of $\sim 1000 \mathrm{deg}^{2}$ with an effective angular resolution of 12 '.8, allowing for constraints on primordial gravitational waves and high signal-to-noise measurements of the $E$-modes across three acoustic peaks. The data reduction was performed using two independent analysis pipelines, one based on a pseudo- $C_{\ell}$ (PCL) cross-correlation approach, and the other on a maximum-likelihood (ML) approach. All data selection criteria and filters were modified until a predefined set of null tests had been satisfied before inspecting any non-null power spectrum. The results derived by the two pipelines are in good agreement. We characterize the $E E, E B$, and $B B$ power spectra between $\ell=25$ and 975 and find that the $E E$ spectrum is consistent with $\Lambda$ CDM, while the $B B$ power spectrum is consistent with zero. Based on these measurements, we constrain the tensor-to-scalar ratio to $r=1.1_{-0.8}^{+0.9}(r<2.8$ at $95 \%$ C.L. $)$ as derived by the ML pipeline, and $r=1.2_{-0.8}^{+0.9}(r<2.7$ at $95 \%$ C.L. $)$ as derived by the PCL pipeline. In one of the fields, we find a correlation with the dust component of the Planck Sky Model, though the corresponding excess power is small compared to statistical errors. Finally, we derive limits on all known systematic errors, and demonstrate that these correspond to a tensor-to-scalar ratio smaller than $r=0.01$, the lowest level yet reported in the literature.
\end{abstract}

Key words: cosmic background radiation - cosmology: observations - gravitational waves - inflation polarization

Online-only material: color figures 


\section{INTRODUCTION}

The theory of inflation explains several well-observed properties of the universe (e.g., Liddle \& Lyth 2000, and references therein): the lack of spatial curvature, the absence of relic monopoles from a grand unified theory's broken symmetry, the large-scale correlations that imply a much larger particle horizon than the Big Bang scenario provides without inflation, and the nearly scale-invariant Gaussian fluctuations. Although inflation was developed to explain these known properties of the universe, which are now probed with high precision by recent cosmological observations (Komatsu et al. 2011; Dunkley et al. 2011; Keisler et al. 2011; Anderson et al. 2012; Hicken et al. 2009; Kessler et al. 2009; Rozo et al. 2010), the model also has a new feature: the early, exponential expansion of space generates a stochastic background of gravitational waves. In the near term, polarization measurements of the cosmic microwave background (CMB) present the most promising approach to detect these gravitational waves, which cause an odd-parity $(B$-mode) polarization pattern on angular scales larger than a degree (Seljak \& Zaldarriaga 1997; Kamionkowski et al. 1997). Detection (or non-detection) of these patterns will place strong constraints on the inflation paradigm.

In the slow-roll approximation (for a review see Liddle \& Lyth 2000 ), the $B$-mode intensity is parameterized by the tensor-toscalar ratio $r$, which is related to the energy scale $V$ of inflation by $V \sim(r / 0.01)^{1 / 4} \times 10^{16} \mathrm{GeV}$. For many classes of inflationary models, $r$ can be as large as $0.01 \lesssim r \lesssim 0.1$ (Boyle et al. 2006).

A combination of CMB-temperature-anisotropy measurements, baryon-acoustic-oscillation data, and supernova observations has given the most stringent limit to date, $r \lesssim 0.2$ at 95\% confidence level (C.L.), nearly limited by cosmic variance (Komatsu et al. 2011; Keisler et al. 2011; Dunkley et al. 2011). In order to improve on these constraints significantly, direct observations of $\mathrm{CMB}$ polarization are required. Thus far the best limit from CMB polarization alone is $r<0.72$ at 95\% C.L. (Chiang et al. 2010), while many experiments have observed even-parity patterns (E-modes; Leitch et al. 2005; Montroy et al. 2006; Sievers et al. 2007; Wu et al. 2007; Bischoff et al. 2008; Brown et al. 2009; Larson et al. 2011; QUIET Collaboration et al. 2011). Experiments currently in operation or under construction seek to reach $r \sim 0.01$ as well as to measure the signature of the gravitational lensing (Essinger-Hileman et al. 2009; Niemack et al. 2010; Ogburn et al. 2010; Eimer et al. 2012; Oxley et al. 2004; Sheehy et al. 2010; Benford et al. 2010; Planck Collaboration et al. 2011; O'Brient et al. 2012; Arnold et al. 2010; Crill et al. 2008; McMahon et al. 2009).

The Q/U Imaging ExperimenT (QUIET) observed the CMB from the ground between 2008 October and 2010 December. The observation site was the Chajnantor plateau at an altitude of $5080 \mathrm{~m}$ in the Atacama Desert in Chile. Two different receivers were employed, corresponding to center frequencies of 43 ( $Q$ band) and $95 \mathrm{GHz}$ ( $W$ band). The results of the $43 \mathrm{GHz}$ measurements have been published in QUIET Collaboration et al. (2011) and included a measurement of the $E$-mode power spectrum between $\ell=25$ and 475 and an upper limit on the tensor-to-scalar ratio of $r<2.2$ at 95\% C.L. In this paper, we report measurements of the CMB polarization power spectra for the $95 \mathrm{GHz}$ data. We note that this experiment played the role of a pathfinder, demonstrating that monolithic-microwaveintegrated-circuit (MMIC) arrays are capable of controlling systematic errors and achieving the sensitivity required to reach $r \lesssim 0.01$.
QUIET was led by Bruce Winstein, who died in 2011 February soon after observations were completed. His intellectual and scientific guidance was crucial to the experiment's success.

\section{INSTRUMENT}

In this section, we summarize the salient features of the $95 \mathrm{GHz}$ instrument. For further details, we refer to separate papers (QUIET Collaboration et al. 2011; QUIET Collaboration 2012, hereafter referred to as QUIET 2011 and QUIET 2012, respectively). Additional information on the QUIET instrument is provided in Bischoff (2010), Brizius (2011), Cleary (2010), Kusaka (2010), Monsalve (2010), Newburgh (2010), Newburgh (2012), and Reeves (2012).

The QUIET telescope consists of a $1.4 \mathrm{~m}$ side-fed classical Dragonian antenna that satisfies the Mizuguchi condition (QUIET 2012). The Cosmic Background Imager telescope mount was reused for the QUIET project. It provides threeaxis motion: azimuth, elevation, and rotation about the optical axis, called "deck" rotation (Padin et al. 2002). The $95 \mathrm{GHz}$ receiver comprises 84 polarization-sensitive radiometers and six radiometers with differential-temperature sensitivity. The array sensitivity is $87 \mu \mathrm{K} \sqrt{\mathrm{s}}$ to the CMB polarization. The instantaneous angular resolution is 11.7 in FWHM. The telescope field of view is roughly circular with a diameter of $\sim 8^{\circ}$.

The coherent QUIET radiometers directly measure the Stokes $Q$ and $U$ parameters (QUIET 2011, 2012). The intensity, $I$, is also recorded by the same radiometers, but with significantly higher noise. One of the strengths of the QUIET design is excellent immunity to both $1 / f$ noise from gain fluctuations and instrumental spurious polarization (hereafter $I$-to- $Q / U$ leakage). The median $1 / f$ knee frequency of the radiometers is $10 \mathrm{mHz}$, significantly below the typical scan frequency of $45-100 \mathrm{mHz}$, resulting in a negligible $1 / f$ noise contribution. The fractional $I$-to- $Q / U$ leakages are $0.2 \%$ for the monopole component, $0.4 \%$ for the dipole component, and $0.2 \%$ for the quadrupole component (QUIET 2012).

The receiver and telescope mirrors are surrounded by an absorbing ground screen, eliminating major contributions from the $300 \mathrm{~K}$ ground emission. The upper component of the ground screen was installed in 2010 January and eliminated two localized far sidelobes with intensities $\sim-60 \mathrm{~dB}$ (QUIET 2012), which existed during the first few months of operation (from 2009 August through 2010 January). For the data from the early part of the season, we reject the part where the Sun entered either of these sidelobes. Scan-synchronous signal due to ground emission is projected out of the maps in the analysis (QUIET 2011). Possible remaining effects are estimated as a systematic error (Section 5.3).

\section{OBSERVATIONS}

With the $95 \mathrm{GHz}$ receiver, we observed from 2009 August 12 until 2010 December 22 and accumulated $7426 \mathrm{hr}$ of data. ${ }^{27}$ Of these data, $72 \%$ were spent on CMB observations, $14 \%$ on Galactic fields, ${ }^{28} 13 \%$ on calibration sources, and $1 \%$ on incomplete observations due to obvious instrumental problems

\footnotetext{
27 The instrument was in the nominal CMB observing configuration only between 2009 August 15 and 2010 December 17. Different configurations were used between 2009 August 12 and 15 and between 2010 December 17 and 22 to calibrate and characterize the instrument.

28 The analysis of the Galactic observations is in progress (see Wehus 2012 for preliminary maps), and final results will appear in a future publication.
} 
Table 1

Data-selection Summary

\begin{tabular}{lcccc}
\hline \hline Field & Observed Time & \multicolumn{3}{c}{ Data Percentage } \\
\cline { 3 - 5 } & $(\mathrm{hr})$ & $\mathrm{ML}$ & $\mathrm{PCL}$ & Both \\
\hline CMB-1 & 1855 & 69.7 & 64.4 & 57.7 \\
CMB-2 & 1444 & 73.1 & 67.1 & 61.2 \\
CMB-3 & 1389 & 64.4 & 58.8 & 52.6 \\
CMB-4 & 650 & 72.1 & 65.4 & 60.4 \\
Total & 5337 & 69.5 & 63.5 & 57.6 \\
\hline
\end{tabular}

Notes. Fraction of data selected for each field by each pipeline. The last column shows the fraction simultaneously selected by both pipelines.

such as a lack of telescope motion. We observed $24 \mathrm{hr}^{\text {day }}{ }^{-1}$, except for interruptions due to a variety of factors such as high wind, heavy snow, power outages, and instrumental failures. Our full-season operating efficiency was 63\%. For the CMB measurements, we selected four low-foreground sky fields, denoted CMB-1, 2, 3, and 4 (QUIET 2011). In total, we collected $5337 \mathrm{hr}$ of CMB data with the $95 \mathrm{GHz}$ receiver (Table 1).

Each observation consists of a series of constant-elevation scans, hereafter collectively called a CES. The scans are in the azimuth direction with a half-amplitude of 7.5 on the sky. Diurnal motion of the sky causes the field to drift through the field of view. After the target has drifted $15^{\circ}$ on the sky, we adjust the azimuth and elevation to retrack the field and begin a new CES. Each individual CES thus scans over an area of $\sim 15^{\circ} \times 15^{\circ}$. Due to the field of view of $\sim 8^{\circ}$ and the fact that the sky does not always drift orthogonal to the scan direction, a larger area is observed in practice. The deck angle is changed by $45^{\circ}$ each week, providing a large degree of immunity to spurious $B$-modes induced by $I$-to- $Q / U$ leakage.

\section{CALIBRATION}

The instrument calibration procedure for the $95 \mathrm{GHz}$ observations is similar to that used for the $43 \mathrm{GHz}$ data (QUIET 2011, 2012). The instantaneous beam point-spread function is derived from observations of Taurus A (hereafter Tau A). The resulting beam function has a width of 11.7 FWHM with a small non-Gaussian correction (QUIET 2012). The telescope pointing model is calibrated with a set of astronomical objects: Tau A, Jupiter, RCW 38, the Moon, and the Galactic center. The residual random scatter after applying all pointing corrections is 5'.1 FWHM. To correct for this, we convolve the beam window function with the residual-pointing-scatter term, and obtain an effective point-spread function of 12'.8 FWHM. The detector angles (i.e., the orientations of the polarization responses) are calibrated to 0.5 precision with the combination of Tau A observations for absolute-angle determination and a sparse-wire-grid calibrator (Tajima et al. 2012; QUIET 2012) for relative angle determination. The considerable improvement in the detector-angle precision compared to the previous $43 \mathrm{GHz}$ analysis (QUIET 2011) is due to a more accurate catalog value of Tau A (Aumont et al. 2010) as well as an improved wire grid calibration. Large and small sky dips (elevation nods of $\pm 20^{\circ}$ and $\pm 3^{\circ}$ amplitudes, respectively) modulate loading from atmospheric emission and allow us to measure the fractional $I$-to- $Q / U$ monopole leakage with $0.3 \%$ precision per calibration, while Jupiter measurements are used to measure the higher-order leakage terms (i.e., dipole and quadrupole) and to confirm the sky-dip monopole-leakage results. The detector responsivities are calibrated using Tau A and sky-dip data as well as the measurement using the sparse wire grid. The typical responsivity is found to be $3.1 \mathrm{mV} \mathrm{K}^{-1}$ in antenna temperature units.

\section{DATA ANALYSIS}

The analysis procedure used for the $95 \mathrm{GHz}$ data reduction follows closely the $43 \mathrm{GHz}$ analysis (QUIET 2011), and we refer the reader to this publication as well as recent $\mathrm{PhD}$ theses (Buder 2012; Chinone 2011; Dumoulin 2011; Monsalve 2012; Næss 2012) for full details. We have implemented two independent analysis pipelines, one based on a maximum-likelihood (ML) technique and the other on a pseudo- $C_{\ell}$ (PCL) cross-correlation technique. The most important improvements since the previous publication are, for the ML pipeline, an adaptive filter procedure in which the filter parameters depend on the data quality of the specific data segment, as well as a pseudo- $C_{\ell}$ null-test estimator, allowing for many more null tests; and, for the PCL pipeline, a different and more robust data division for the crosscorrelation, ${ }^{29}$ taking further advantage of the scanning strategy.

The process to extract cosmological results from raw timeordered data (TOD), containing measurements of the Stokes $Q$ and $U$ parameters as well as the telescope pointing information, can be summarized in three steps: TOD pre-processing, map making, and power-spectrum and parameter estimation. The TOD pre-processing involves estimating and applying calibration factors, characterizing the detector noise, and applying high-pass, low-pass, and azimuth filters to minimize the effects of atmospheric fluctuations, far sidelobes, excess highfrequency instrumental noise, and ground pickup. Then, sky maps are generated by projecting the $Q$ and $U$ intensities into Galactic coordinates, taking into account the telescope pointing information, using standard map-making equations (QUIET 2011). Figure 1 shows the ML Stokes $Q$ and $U$ maps of the CMB-1 field generated with the ML pipeline. Powerspectrum estimation is performed with one of two techniques, depending on the pipeline. The ML pipeline implements a standard Newton-Raphson ML solver (Bond et al. 1998), while the PCL pipeline implements the MASTER pseudo- $C_{\ell}$ algorithm (Hivon et al. 2002; Hansen \& Gorski 2003). Prior to powerspectrum estimation, both pipelines mask Centaurus A, and the PCL pipeline also masks Pictor A.

In the following we describe the data selection, analysis validation, and systematic-error assessment. In optimizing the analysis configuration, it is important that the optimization process itself does not introduce experimenter biases, for instance by removing purely statistical fluctuations in the data selection. QUIET is the first CMB experiment to have adopted a strict blind-analysis policy (Klein \& Roodman 2005), in which all data selection criteria, filters, and calibrations are adjusted and finalized, and the systematic errors are assessed prior to looking at any cosmological power spectrum. This process was described in detail in QUIET 2011, and we have adopted the same policy for the $95 \mathrm{GHz}$ analysis.

\subsection{Data Selection}

Each QUIET radiometer provides four output channels ("detector diodes"), resulting in a total of 336 output channels from 84 polarization-sensitive radiometers. We use 308 good channels for analysis (QUIET 2012). Starting from the resulting data for all CESs, we define two different classes of data selection

\footnotetext{
29 We cross-correlate among 40 subsets of data. Each subset corresponds to a specific boresight azimuth and deck range. There are five azimuth and eight deck ranges.
} 

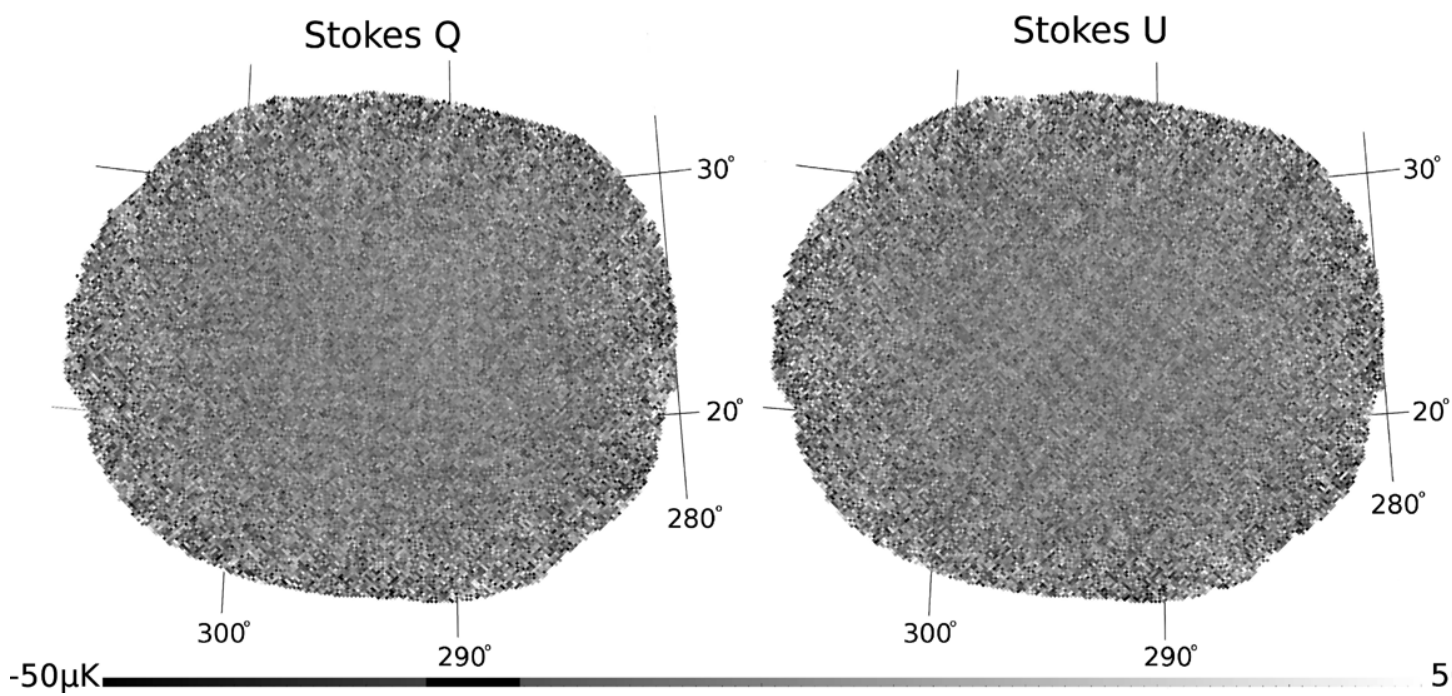

Figure 1. QUIET CMB polarization maps of the CMB-1 field in Galactic coordinates at $95 \mathrm{GHz}$. The left (right) panel shows Stokes $Q(U)$, where the polarization angle is defined with respect to the Galactic North Pole. Note the coherent vertical/horizontal patterns in the $Q$ map, and the diagonal patterns in the $U$ map; these are the expected signature of a pure $E$-mode signal. No filtering has been applied to this map beyond subtracting the very largest angular scales $(\ell<25)$, to which QUIET is not sensitive.

criteria. In the first class, we impose criteria that select or reject an entire CES. These include the criteria based on atmospheric conditions, instrument malfunctions, or unusual conditions for the temperature regulation in the focal plane. In the second class, we apply selection criteria to individual detector diodes in each CES (CES-diodes). For instance, a CES-diode is rejected if (1) the measured noise properties show poor agreement with the noise model, (2) the $1 / f$ knee frequency is anomalously high, (3) the white-noise level is non-stationary, (4) there are glitches in the time domain or strong spikes in the Fourier domain, or (5) there is evidence of a large scan-synchronous signal.

Table 1 lists the fractions of data that satisfy the criteria and are used for map making and power-spectrum estimation.

\subsection{Analysis Validation}

Having defined our data selection criteria and filters, we need to validate the accepted data set and analysis parameters. ${ }^{30}$ Our most valuable tool for this is a so-called null-test suite (QUIET 2011). In each null test, the full data are split into two subsets. From these, we make individual sky maps, $m_{1}$ and $m_{2}$, as well as the corresponding difference map, $m_{\text {diff }} \equiv\left(m_{1}-m_{2}\right) / 2$. By design, the true sky signal cancels in this map, and the result should be consistent with noise. We therefore compute the $E E$ and $B B$ power spectra of this map, and check for consistency with the zero-signal hypothesis by comparing to simulations. In the current analysis, the null suite consists of 32 and 23 tests for the PCL and ML pipelines, respectively, with each test targeting a possible source of signal contamination or miscalibration. These are selected to be highly independent; a statistical correlation between null power spectra of two different null-test divisions is typically 0.05 .

For each power-spectrum bin $b$, we calculate the statistic $\chi_{\text {null }}(b) \equiv C_{b}^{\text {null }} / \sigma_{b}$, where $C_{b}^{\text {null }}$ is the observed difference power spectrum and $\sigma_{b}$ is a Monte Carlo (MC) based estimate of the corresponding standard deviation. We evaluate both $\chi_{\text {null }}$ and its

\footnotetext{
30 Note that the data selection criteria are improved through an iterative process of applying the analysis-validation metrics. In the current analysis, $\sim 50$ different configurations were considered before reaching the final configuration.
}

Table 2

Validation-test Summary

\begin{tabular}{|c|c|c|c|c|c|c|}
\hline \multirow[t]{2}{*}{ Field } & \multicolumn{2}{|c|}{ Mean of $\chi_{\text {null }}$} & \multicolumn{2}{|c|}{ Total $\chi_{\text {null }}^{2}$} & \multicolumn{2}{|c|}{$\chi_{\text {null }}^{2}$ Outlier } \\
\hline & ML & PCL & ML & PCL & ML & PCL \\
\hline CMB-1 & 0.40 & 0.26 & 0.31 & 0.78 & 0.63 & 0.14 \\
\hline CMB-2 & 0.54 & 0.46 & 0.08 & 0.06 & 0.02 & 0.40 \\
\hline CMB-3 & 0.42 & 0.74 & 0.31 & 0.50 & 0.72 & 0.45 \\
\hline CMB-4 & 0.06 & 0.08 & 0.43 & 0.76 & 0.80 & 0.18 \\
\hline
\end{tabular}

Notes. Results of the three predefined validation tests using the mean of $\chi_{\text {null }}$, the sum of $\chi_{\text {null }}^{2}$, and the worst outlier of $\chi_{\text {null }}^{2}$. All values are PTEs defined such that a large deviation from zero results in a small PTE.

square for all $b ; \chi_{\text {null }}$ is sensitive to systematic biases in the null spectra, while $\chi_{\text {null }}^{2}$ is more responsive to outliers.

Prior to the analysis, we defined three critical tests that had to be passed before continuing to cosmological analysis, based on (1) the mean value of $\chi_{\text {null }}$, (2) the sum of $\chi_{\text {null }}^{2}$, and (3) the maximum of $\chi_{\text {null }}^{2}$, all computed including the entire suite of $E E$ and $B B$ null power spectra. A given analysis configuration passes when these statistics are consistent with the null hypothesis. Table 2 lists the probabilities to exceed (PTE) for the final configuration, and Figure 2 shows the PTE distribution of $\chi_{\text {null }}^{2}$. The PTEs are defined such that a large deviation from zero results in a low PTE. This corresponds to two-sided PTEs for the mean of $\chi_{\text {null }}$ and one-sided PTEs for the total $\chi_{\text {null }}^{2}$ and the $\chi_{\text {null }}^{2}$ outlier. The mean of the $\chi_{\text {null }}$ distributions over all fields is $-0.018 \pm 0.015$ for the PCL pipeline and $0.003 \pm 0.017$ for the ML pipeline. We do not detect any bias with our final analysis configuration.

We also generate 1000 random null divisions and compare the widths of the resulting $\chi_{\text {null }}$ distributions between data and MCs using the PCL pipeline. We find these to be consistent, and we verify our estimate of the statistical uncertainty in each multipole bin with a precision of $3.1 \%$. Finally, we evaluate the differences of non-null spectra among the fields, before looking at individual non-null spectra. These differences are consistent with the hypothesis of statistical isotropy (i.e., each field has the same underlying power spectrum), with a PTE of 0.15. 


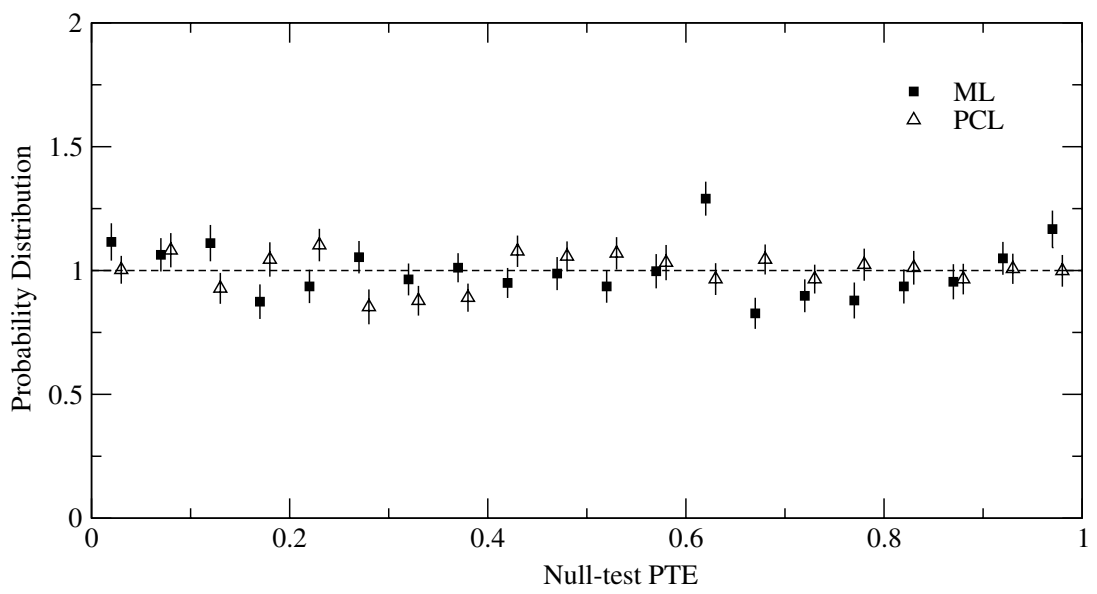

Figure 2. Null-test-PTE distributions for $\chi_{\text {null }}^{2}$ for both the ML and PCL pipelines. Each is consistent with the uniform expectation.

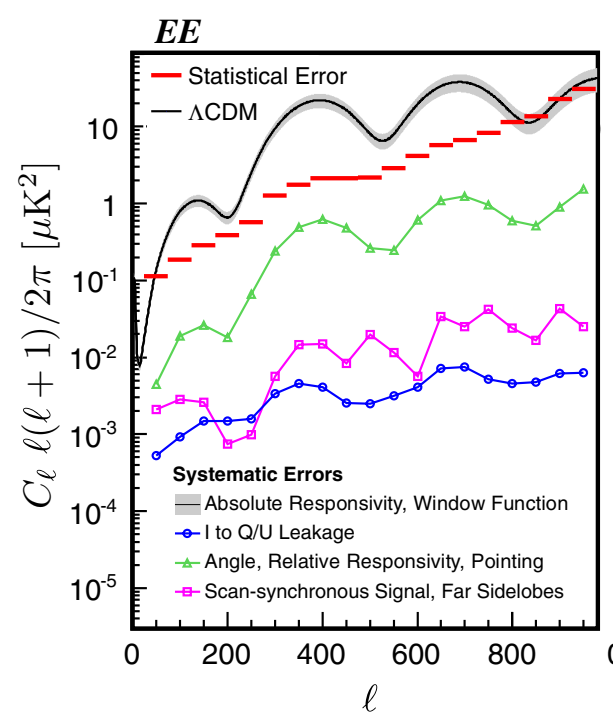

\section{$B B$}

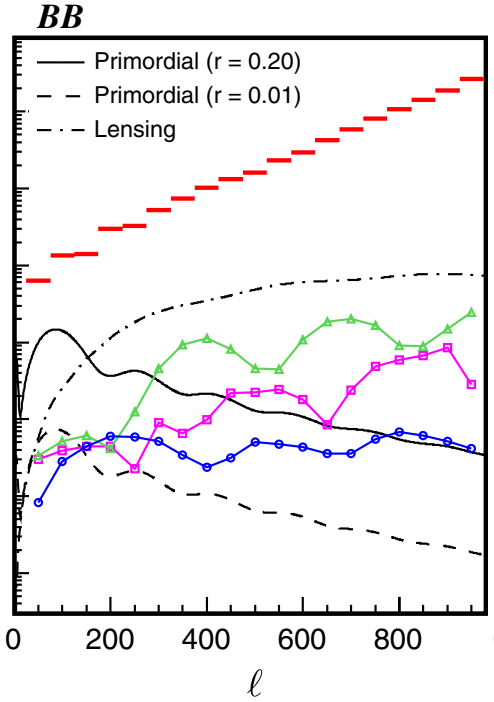

$\boldsymbol{E B}$

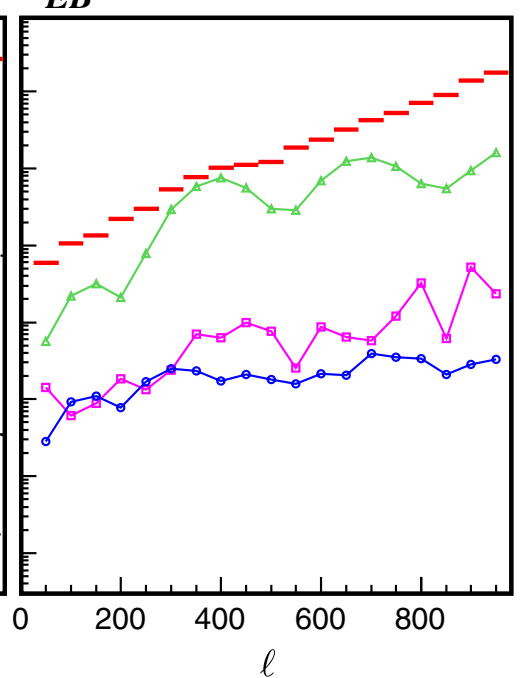

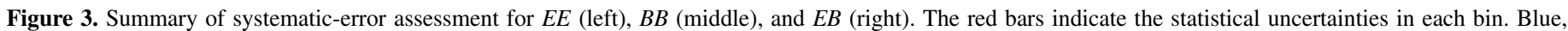

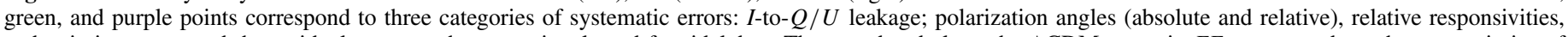

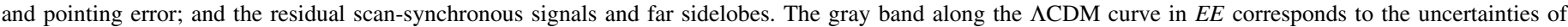

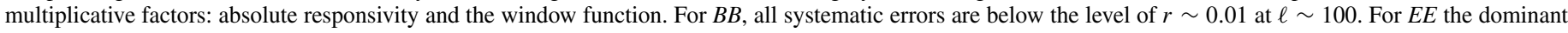

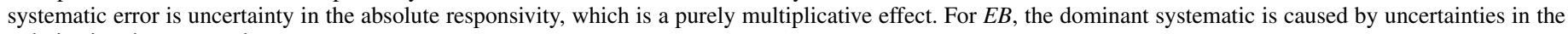
polarization detector angle.

(A color version of this figure is available in the online journal.)

\subsection{Systematic Errors}

We study the contributions from instrumental systematic errors using the methodology of QUIET 2011. The main effects considered are (1) uncertainties in absolute responsivity and the window function; (2) $I$-to- $Q / U$ leakage; (3) uncertainties in polarization angles, relative responsivities, and pointing; and (4) residual contamination from scan-synchronous signals and far sidelobes. In each case, we set up an empirical model of the systematic effect and propagate this through the PCL pipeline. The results from these calculations are summarized in Figure 3.

The most important conclusion is that the systematic errors in the $B B$ spectrum are very small. For the multipole range relevant for estimation of the tensor-to-scalar ratio, $\ell \sim 100$, each effect is smaller than or comparable to the signal corresponding to $r \sim 0.01$, the lowest level ever reported in the literature. It is also noteworthy that this limit improves on that reported for the $43 \mathrm{GHz}$ data $(r<0.1$; QUIET 2011) by an order of magnitude. This is due to improved rejection of $I$-to- $Q / U$ leakage, better detector-angle calibration, and lower levels of sidelobe contamination resulting from the installation of the upper parts of the ground screen.

For the $E E$ power spectrum, the systematic-error budget is dominated by uncertainties in the multiplicative responsivity calibration. The total uncertainty is $8 \%$, almost equally contributed from three dominant sources: the uncertainty of the polarization flux of Tau A (5\%; Weiland et al. 2011), the uncertainty in the beam solid angle (5\%), and the uncertainty associated with modeling the time variation and relative responsivity among the detector channels (4\%). This translates into an uncertainty of $17 \%$ in the power spectrum. For comparison, the statistical uncertainty in the $E E$ spectrum is about $8 \%$ of the central value at its minimum around $\ell \sim 400$. It is important to note that the responsivity effect is purely multiplicative and therefore cannot create spurious $B$-mode signal. The uncertainty of the window function is another multiplicative factor highly correlated among different $\ell$ bins, with the magnitude 
Table 3

QUIET Polarization Power Spectra

\begin{tabular}{|c|c|c|c|c|c|c|}
\hline \multirow[b]{2}{*}{$\ell_{\min }$} & \multirow[b]{2}{*}{$\ell_{\max }$} & \multicolumn{2}{|c|}{ ML Pipeline } & \multicolumn{2}{|c|}{ PCL Pipeline } & \multirow{2}{*}{$\begin{array}{c}\text { Syst. } \\
\text { Window Functior }\end{array}$} \\
\hline & & $E E$ & $E E / q$ & $E E$ & $E E / q$ & \\
\hline 26 & 75 & $0.28_{-0.14}^{+0.17}$ & $0.21_{-0.11}^{+0.13}$ & $0.29_{-0.10}^{+0.13}$ & $0.24_{-0.08}^{+0.11}$ & 0.00 \\
\hline 76 & 125 & $1.06_{-0.21}^{+0.24}$ & $0.78_{-0.16}^{+0.18}$ & $0.82_{-0.17}^{+0.20}$ & $0.67_{-0.14}^{+0.16}$ & 0.01 \\
\hline 126 & 175 & $1.80_{-0.32}^{+0.35}$ & $1.34_{-0.23}^{+0.26}$ & $1.35_{-0.27}^{+0.30}$ & $1.11_{-0.22}^{+0.25}$ & 0.01 \\
\hline 176 & 225 & $0.93_{-0.33}^{+0.37}$ & $0.69_{-0.25}^{+0.28}$ & $1.48_{-0.37}^{+0.41}$ & $1.21_{-0.30}^{+0.34}$ & 0.02 \\
\hline 226 & 275 & $3.5_{-0.6}^{+0.7}$ & $2.6_{-0.4}^{+0.5}$ & $2.9_{-0.5}^{+0.6}$ & $2.3_{-0.5}^{+0.5}$ & 0.02 \\
\hline 276 & 325 & $13.5_{-1.3}^{+1.3}$ & $10.0_{-1.0}^{+1.0}$ & $12.7_{-1.2}^{+1.3}$ & $10.4_{-1.0}^{+1.1}$ & 0.03 \\
\hline 326 & 375 & $24.8_{-2.1}^{+2.0}$ & $18.3_{-1.5}^{+1.5}$ & $21.1_{-1.7}^{+1.8}$ & $17.3_{-1.4}^{+1.5}$ & 0.04 \\
\hline 376 & 425 & $30.6_{-2.4}^{+2.6}$ & $22.7_{-1.8}^{+1.9}$ & $27.2_{-2.1}^{+2.2}$ & $22.3_{-1.7}^{+1.8}$ & 0.05 \\
\hline 426 & 475 & $19.8_{-2.4}^{+2.3}$ & $14.7_{-1.8}^{+1.7}$ & $18.3_{-2.1}^{+2.2}$ & $15.0_{-1.7}^{+1.8}$ & 0.05 \\
\hline 476 & 525 & $15.5_{-2.6}^{+2.7}$ & $11.5_{-1.9}^{+2.0}$ & $9.1_{-2.1}^{+2.3}$ & $7.4_{-1.7}^{+1.9}$ & 0.06 \\
\hline 526 & 575 & $14.1_{-3.1}^{+3.3}$ & $10.5_{-2.3}^{+2.4}$ & $11.4_{-2.8}^{+3.0}$ & $9.3_{-2.3}^{+2.5}$ & 0.07 \\
\hline 576 & 625 & $24.7_{-4.3}^{+4.5}$ & $18.3_{-3.2}^{+3.3}$ & $19.5_{-4.0}^{+4.2}$ & $16.0_{-3.3}^{+3.5}$ & 0.08 \\
\hline 626 & 675 & $49_{-7}^{+7}$ & $36_{-5}^{+5}$ & $40_{-6}^{+6}$ & $33_{-5}^{+5}$ & 0.09 \\
\hline 676 & 725 & $40_{-8}^{+8}$ & $30_{-6}^{+6}$ & $37_{-7}^{+7}$ & $31_{-5}^{+6}$ & 0.10 \\
\hline 726 & 775 & $25_{-10}^{+10}$ & $18_{-7}^{+7}$ & $21_{-8}^{+8}$ & $17_{-7}^{+7}$ & 0.11 \\
\hline 776 & 825 & $20_{-13}^{+14}$ & $15_{-10}^{+10}$ & $30_{-11}^{+12}$ & $25_{-9}^{+10}$ & 0.12 \\
\hline 826 & 875 & $-9_{-16}^{+17}$ & $-7_{-12}^{+13}$ & $-8_{-13}^{+14}$ & $-7_{-11}^{+11}$ & 0.14 \\
\hline 876 & 925 & $93_{-28}^{+29}$ & $69_{-21}^{+21}$ & $68_{-22}^{+24}$ & $55_{-18}^{+19}$ & 0.15 \\
\hline \multirow[t]{2}{*}{926} & 975 & $35_{-37}^{+39}$ & $26_{-27}^{+29}$ & $85_{-30}^{+32}$ & $69_{-25}^{+26}$ & 0.17 \\
\hline & & \multicolumn{2}{|c|}{$q=1.35 \pm 0.05_{-0.22}^{+0.26}$} & \multicolumn{2}{|c|}{$q=1.22 \pm 0.04_{-0.17}^{+0.22}$} & \\
\hline$\ell_{\min }$ & $\ell_{\max }$ & $B B(95 \% \mathrm{UL})$ & $E B$ & $B B(95 \% \mathrm{UL})$ & $E B$ & $E B$ syst. \\
\hline 26 & 75 & $-0.08_{-0.08}^{+0.10}(0.19)$ & $0.07_{-0.04}^{+0.03}$ & $0.03_{-0.05}^{+0.07}(0.18)$ & $-0.04_{-0.04}^{+0.04}$ & \pm 0.01 \\
\hline 76 & 125 & $0.26_{-0.14}^{+0.15}(0.55)$ & $-0.04_{-0.11}^{+0.11}$ & $0.24_{-0.12}^{+0.15}(0.50)$ & $0.03_{-0.11}^{+0.11}$ & \pm 0.02 \\
\hline 126 & 175 & $-0.25_{-0.14}^{+0.16}(0.23)$ & $0.12_{-0.16}^{+0.16}$ & $-0.22_{-0.13}^{+0.15}(0.23)$ & $0.17_{-0.14}^{+0.15}$ & \pm 0.03 \\
\hline 176 & 225 & $0.53_{-0.28}^{+0.31}(1.09)$ & $0.13_{-0.22}^{+0.22}$ & $0.39_{-0.28}^{+0.32}(0.95)$ & $0.17_{-0.23}^{+0.24}$ & \pm 0.02 \\
\hline 226 & 275 & $-0.59_{-0.34}^{+0.37}(0.52)$ & $0.11_{-0.34}^{+0.33}$ & $-0.60_{-0.31}^{+0.35}(0.49)$ & $0.12_{-0.32}^{+0.33}$ & \pm 0.08 \\
\hline 276 & 325 & $-0.4_{-0.5}^{+0.6}(1.0)$ & $-0.1_{-0.6}^{+0.6}$ & $-0.2_{-0.5}^{+0.5}(1.0)$ & $0.1_{-0.6}^{+0.6}$ & \pm 0.29 \\
\hline 326 & 375 & $-0.5_{-0.7}^{+0.7}(1.2)$ & $2.3_{-0.9}^{+0.8}$ & $-0.1_{-0.7}^{+0.8}(1.5)$ & $2.0_{-0.8}^{+0.9}$ & \pm 0.59 \\
\hline 376 & 425 & $-0.1_{-1.0}^{+1.0}(2.1)$ & $1.2_{-1.1}^{+1.1}$ & $0.4_{-1.0}^{+1.1}(2.5)$ & $0.9_{-1.1}^{+1.1}$ & \pm 0.75 \\
\hline 426 & 475 & $1.6_{-1.4}^{+1.5}(4.4)$ & $1.8_{-1.3}^{+1.3}$ & $1.2_{-1.3}^{+1.4}(3.7)$ & $2.5_{-1.2}^{+1.2}$ & \pm 0.55 \\
\hline 476 & 525 & $-0.1_{-1.8}^{+1.9}(4.0)$ & $0.9_{-1.6}^{+1.6}$ & $-2.5_{-1.6}^{+1.7}(2.3)$ & $0.2_{-1.3}^{+1.4}$ & \pm 0.30 \\
\hline 526 & 575 & $4.2_{-2.7}^{+2.8}(9.2)$ & $0.3_{-2.0}^{+2.0}$ & $2.3_{-2.3}^{+2.4}(6.6)$ & $-1.6_{-2.0}^{+1.9}$ & \pm 0.28 \\
\hline 576 & 625 & $3.8_{-3.5}^{+3.7}(10.5)$ & $3.4_{-2.8}^{+2.7}$ & $-1.1_{-2.9}^{+3.1}(5.6)$ & $1.8_{-2.5}^{+2.6}$ & \pm 0.70 \\
\hline 626 & 675 & $9.2_{-4.9}^{+5.2}(18.3)$ & $7.8_{-4.0}^{+4.1}$ & $6.3_{-4.1}^{+4.4}(13.8)$ & $4.8_{-3.5}^{+3.5}$ & \pm 1.25 \\
\hline 676 & 725 & $3_{-6}^{+7}(16)$ & $1_{-5}^{+5}$ & $8_{-6}^{+6}(18)$ & $6.7_{-4.8}^{+4.8}$ & \pm 1.40 \\
\hline 726 & 775 & $-8_{-8}^{+9}(13)$ & $3_{-6}^{+6}$ & $1_{-8}^{+8}(17)$ & $-11_{-6}^{+5}$ & \pm 1.06 \\
\hline 776 & 825 & $7_{-12}^{+13}(31)$ & $-19_{-9}^{+9}$ & $5_{-10}^{+11}(26)$ & $-14_{-8}^{+8}$ & \pm 0.68 \\
\hline 826 & 875 & $10_{-17}^{+18}(44)$ & $15_{-12}^{+12}$ & $2_{-14}^{+15}(31)$ & $-9_{-10}^{+10}$ & \pm 0.55 \\
\hline 876 & 925 & $46_{-26}^{+27}(93)$ & $4_{-19}^{+19}$ & $-3_{-18}^{+19}(37)$ & $0_{-15}^{+15}$ & \pm 0.93 \\
\hline 926 & 975 & $-52_{-33}^{+35}(44)$ & $0_{-25}^{+25}$ & $-28_{-26}^{+27}(41)$ & $17_{-19}^{+19}$ & \pm 1.59 \\
\hline
\end{tabular}

Notes. Tabulated values are given in CMB thermodynamic units of $\mu \mathrm{K}^{2}$, scaled as $C_{\ell} \ell(\ell+1) / 2 \pi$. We present the results from both the ML and PCL pipelines; they are in excellent statistical agreement. The column $E E / q$ shows the $E E$ power spectrum normalized to $q=1$, as plotted in Figure 5. The fit value of $q$ is also shown in the table, where the first and second errors are statistical and systematic, respectively. The column of $B B-$ power-spectrum values also provides $95 \%$ confidence level upper limits in parentheses. We also list two relevant systematic-error contributions besides the uncertainty from the responsivity calibration: the fractional error due to the uncertainty of the beam window function, and the total systematic error in the $E B$ power spectrum in units of $\mu \mathrm{K}^{2}$. Note that they are both highly correlated among $\ell$ bins. We assume the $\Lambda$ CDM prediction (i.e., $q=1$ ) for the $C_{\ell}^{E E}$ spectrum sourcing the systematic error in the $E B$ power; the $E B$ systematic-error estimate should be multiplied by the fit value of $q$ to directly compare with the presented $E B$ power spectrum.

dependent on $\ell$. The uncertainty comes from both the beam window function and the smearing factor due to the pointing error, and is listed in Table 3. These errors are smaller than the EE statistical uncertainties.
The dominant systematic uncertainty for $E B$ is due to calibration errors in the detector polarization angles. To first approximation, an error in the absolute-polarization-angle calibration of $\delta \psi$ induces a spurious $E B$ spectrum proportional 
to $\sim C_{\ell}^{E E} \sin 2 \delta \psi$, and a $B B$ spectrum proportional to $\sim C_{\ell}^{E E} \sin ^{2} 2 \delta \psi$. Uncertainties in the relative polarization angles among detectors contribute to the systematic errors in $E B$ and $B B$ spectra in a similar manner. The calculations summarized in Figure 3 capture both these effects through simulations based on the $\Lambda C D M$ prediction for $C_{\ell}^{E E}$. As seen in this figure, these polarization-angle uncertainties lead to systematic errors almost as large as the statistical errors for $E B$ around $\ell \sim 400$, while for $B B$ they are small everywhere and comparable to other sources of systematic errors. Table 3 lists the total systematic error for the $E B$ power spectrum.

\section{POWER SPECTRA AND COSMOLOGICAL PARAMETERS}

The measurements of the $E E, E B$, and $B B$ power spectra are tabulated in Table 3, and plotted in Figure 4. The $E E$ spectrum is strongly signal dominated up to $\ell \sim 800$, and three acoustic peaks are clearly traced. Both the $B B$ and $E B$ spectra are consistent with zero within the estimated statistical and systematic uncertainties. The dominant $E E$ power is also visible in the maps shown in Figure 1. Note that these maps have not been filtered, except by subtracting the very largest scales $(\ell \lesssim 25$ ), to which QUIET is not sensitive. One can see a distinct vertical-horizontal coherent pattern on small angular scales in the Stokes $Q$ map, and a similar diagonal pattern in the $U$ map. This is the expected signature of an $E$-mode signal.

The results from the two pipelines are consistent with each other. The most noticeable difference is a single overall multiplicative factor, which is only relevant in evaluating the consistency of the $E E$ power spectra. This factor comes from different responsivity modeling and is consistent with the systematicerror budget discussed in Section 5.3.

When assessing the consistency of the $E E$ power spectrum with the $\Lambda C D M$ prediction, it is convenient to factor the spectrum measurement into an overall amplitude and the spectral shape of the acoustic peaks. We fit a free amplitude, $q$, relative to the $E E$ spectrum predicted by the best-fit seven-year WMAP $\Lambda \mathrm{CDM}$ parameters (Komatsu et al. 2011) to the spectrum from each pipeline, and find $q=1.22 \pm 0.04$ (stat) ${ }_{-0.17}^{+0.22}$ (syst) and $q=1.35 \pm 0.05$ (stat) ${ }_{-0.22}^{+0.26}$ (syst) for the PCL and ML pipelines, respectively. These values are consistent with the $\Lambda$ CDM prediction of $q=1$, and correspond to PTEs of 0.20 and 0.06 , respectively. Figure 5 provides a spectral shape comparison. Here we see that the measured $E E$ spectrum rescaled to $q=1$ accurately traces the first three acoustic peaks predicted by the $\Lambda \mathrm{CDM}$ model. We assess the overall consistency with the $\Lambda$ CDM hypothesis by calculating a total $\chi^{2}$ relative to $\Lambda$ CDM (and relative to $C_{\ell}^{B B}=C_{\ell}^{E B}=0$ ), taking into account the systematic uncertainties due to the responsivity calibration in $E E$ and the systematic error in $E B$ primarily due to detector polarization angles. The former is incorporated by introducing a nuisance parameter for the absolute responsivity constrained by a Gaussian distribution with a standard deviation equal to the assigned systematic error. The latter is incorporated by modeling the $E B$ systematic error as $s C_{\ell}^{E B \text {,syst }}$, where the scale factor $s$ is constrained by a Gaussian with $\sigma=1$ and $C_{\ell}^{E B \text {,syst }}$ is the systematic error estimated in Section 5.3; this means we assume the systematic errors are completely correlated among different $\ell$ bins. The systematic errors in $B B$ are negligibly small. Including the systematic-error contributions, we find $\chi^{2}$ of 67.3 and 67.9 for the PCL and ML pipelines, respectively. With 57 degrees of

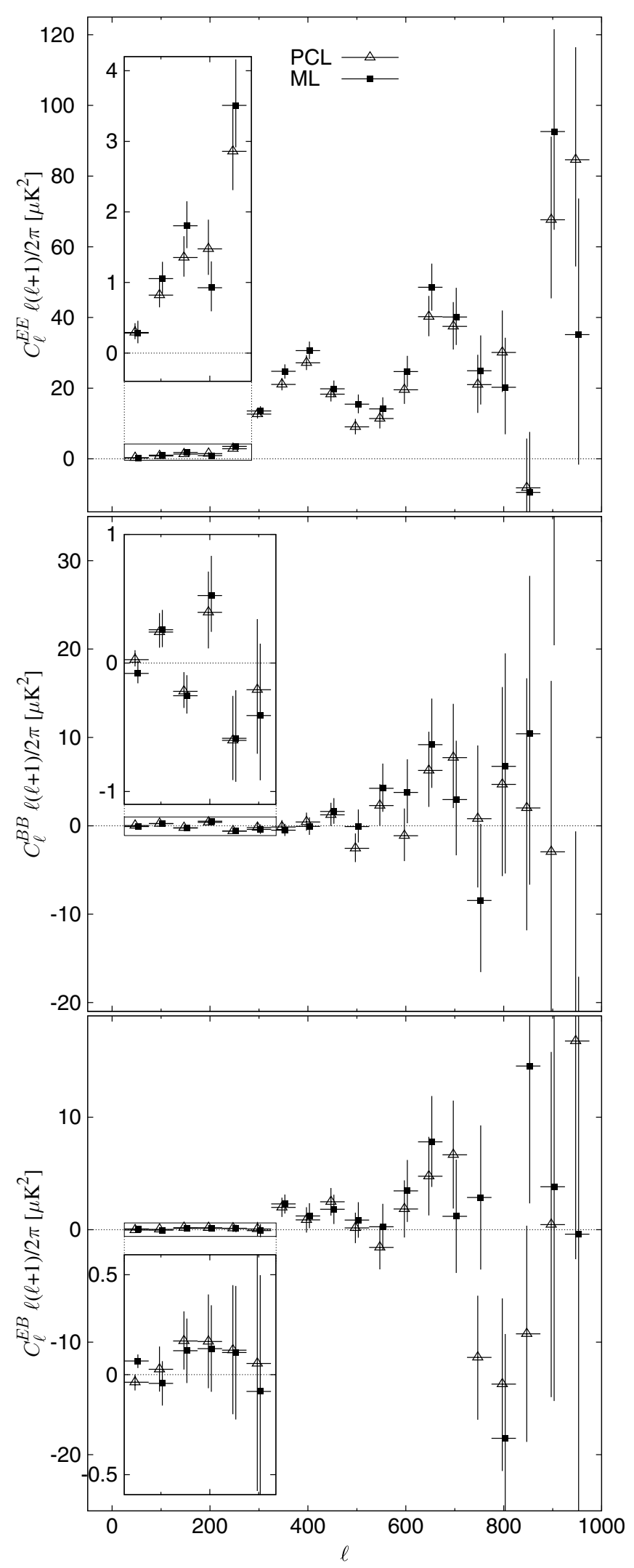

Figure 4. QUIET $95 \mathrm{GHz}$ power spectra, co-added over all four CMB fields. The panels show the $E E$ (top), $B B$ (middle), and $E B$ (bottom) spectra, and the insets show the low- $\ell$ region in detail. The central $\ell$ values from the two pipelines are slightly offset for display purposes. Note that the error bars indicate statistical errors only; see Section 5.3 for a discussion of systematic errors. Typical correlations among neighboring bins are $\sim-0.1$. The full set of three spectra is consistent with the $E E$ spectrum predicted by the $\Lambda C D M$ model and $C_{\ell}^{B B}=C_{\ell}^{E B}=0$. 


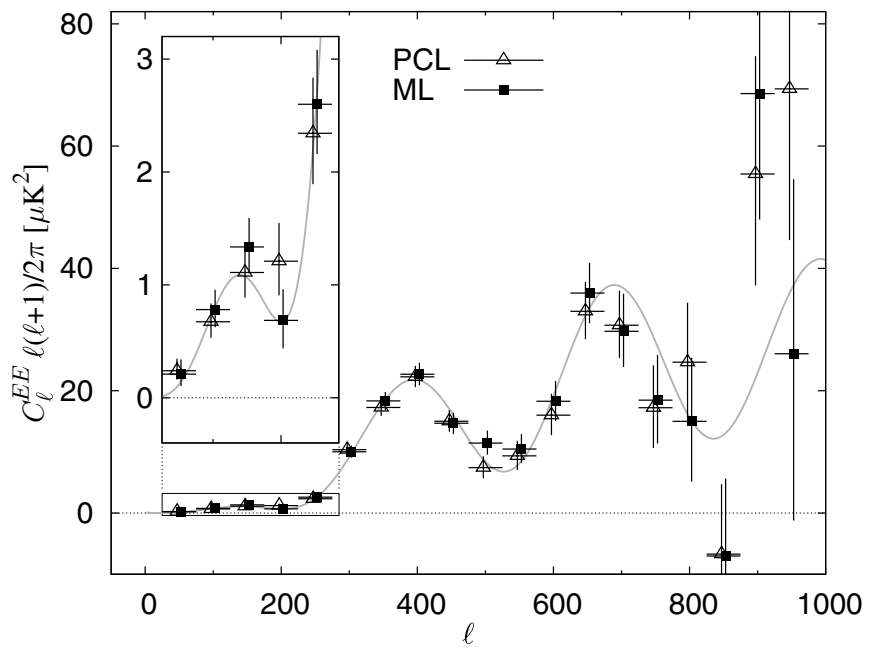

Figure 5. Comparison of the QUIET $E E$ spectrum with current best-fit $\Lambda \mathrm{CDM}$ model after scaling the absolute responsivity to $q=1$. The central $\ell$ values from the two pipelines are slightly offset for display purposes. Typical correlations among neighboring bins are $\sim-0.1$. The results from the two pipelines are consistent, and the shape of the QUIET EE spectrum is in excellent agreement with the $\Lambda$ CDM model. freedom, these values correspond to PTEs of 0.16 and 0.15 , respectively; the derived spectra are consistent with $\Lambda \mathrm{CDM}$.

Since we find no significant excess in the $B B$ power spectrum, we place an upper limit on possible $B B$ power in each bin. The ML pipeline calculates the upper limit by the $95 \%$ integral of the positive part of the likelihood, while the PCL pipeline adopts a frequentist-based hypothesis-testing method. Specifically, the upper limit $\mu$ is defined by $0.05=p\left(q_{\mu}>q_{\mu}^{\text {obs }} \mid \mu\right) / p\left(q_{\mu}>\right.$ $\left.q_{\mu}^{\text {obs }} \mid 0\right)$, where $p(\cdots \mid \mu)$ and $p(\cdots \mid 0)$ represent $p$-values of the null hypothesis with power $\mu$ and an alternative hypothesis with zero power, respectively. The parameters $\mu, q_{\mu}$, and $q_{\mu}^{\text {obs }}$ correspond to the bandpower $C_{b}$, the test statistic for upper limit defined in Cowan et al. (2011), and the test statistic $q_{\mu}$ calculated for the observed bandpower $\hat{C}_{b}$, respectively. Table 3 lists the derived upper limits.

We constrain the tensor-to-scalar ratio $r$ using standard likelihood methods and including only the $B B$ spectrum at low multipoles $(26 \leqslant \ell \leqslant 175)$. For simplicity, we consider only the amplitude of a $B B$ template computed with the standard $\Lambda \mathrm{CDM}$ concordance parameters, and fix the tensor spectral index to $n_{t}=0$ (QUIET 2011; Chiang et al. 2010). In constraining $r$, the uncertainty of the responsivity calibration is eliminated by simultaneously fitting $E E$ and $B B$ power spectra using the $\Lambda \mathrm{CDM}$ templates. We define the fit function as $C_{\ell}^{E E}(q)=$ $q C_{\ell}^{E E \text {,fid }}$ and $C_{\ell}^{B B}(q, r)=r q C_{\ell}^{B B \text {,fid }}$. Here $C_{\ell}^{E E \text {,fid }}$ and $C_{\ell}^{B B \text {,fid }}$ denote the fiducial $\Lambda C D M E E$-power-spectrum template and

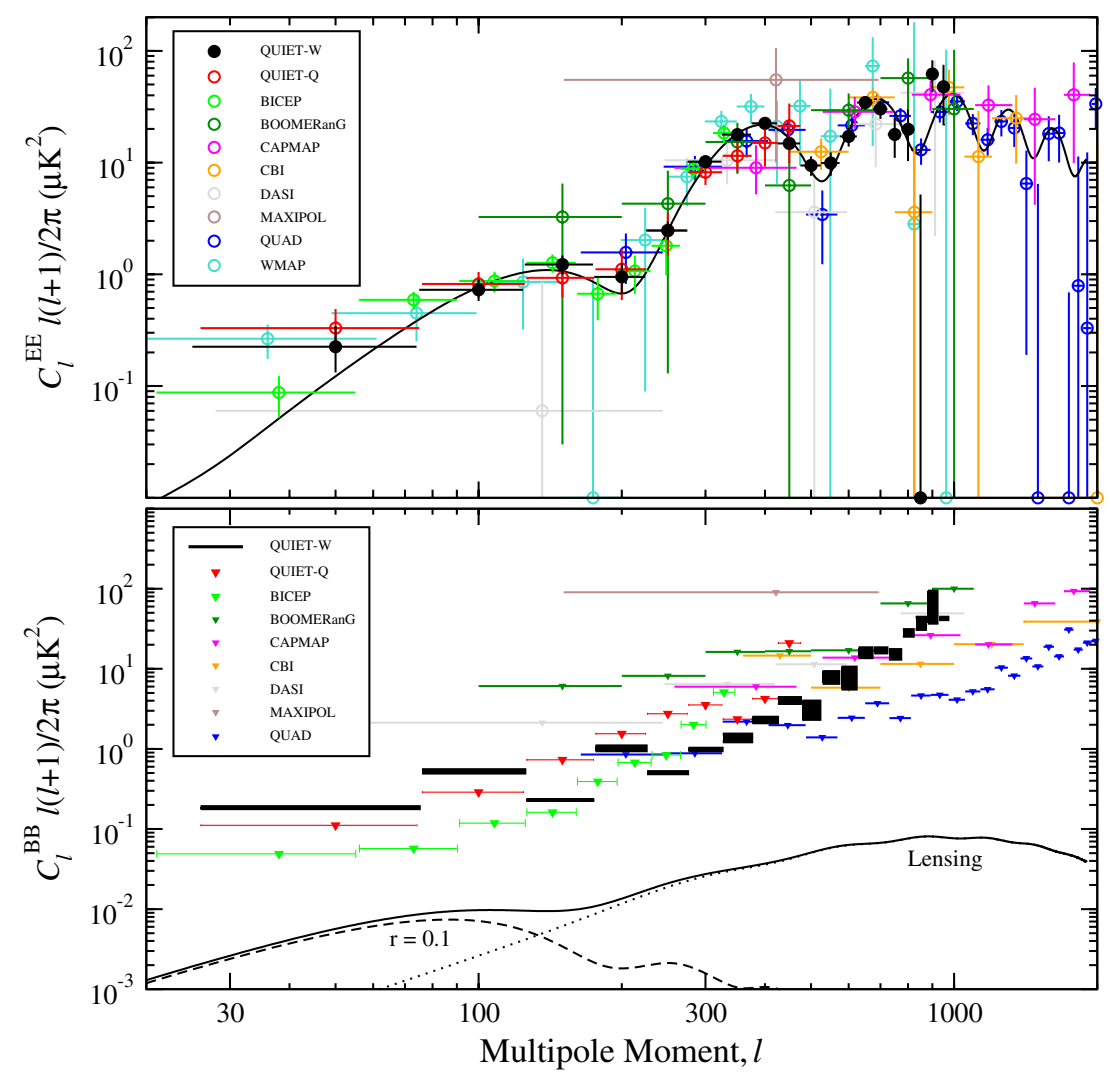

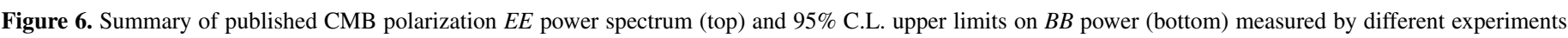

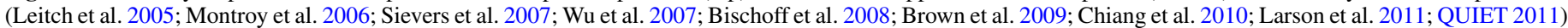

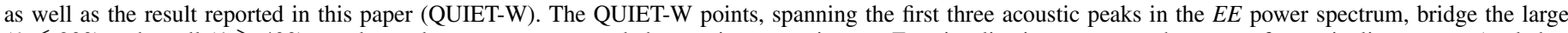

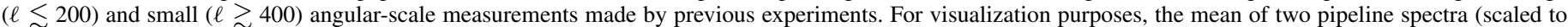

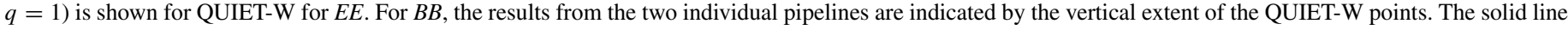

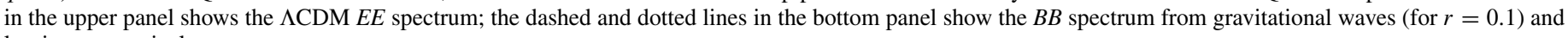
lensing, respectively.

(A color version of this figure is available in the online journal.) 
the $B B$-power-spectrum template with $r=1$ and $n_{t}=0$, respectively. Note that this does not imply that we use $E E$ power to constrain the tensor modes, as the $\Lambda \mathrm{CDM} E E$ template only contains the scalar contribution. This method exploits the fact that $r$ is by definition a ratio and does not depend on the common overall scaling factor. From the simultaneous fit, the ML pipeline finds $r=1.1_{-0.8}^{+0.9}$, with a $95 \%$ C.L. upper limit of $r<2.8$, and the PCL pipeline finds $r=1.2_{-0.8}^{+0.9}$, corresponding to an upper limit of $r<2.7$. The systematic uncertainty is negligible, at the level of $r=0.01$.

\section{FOREGROUNDS}

We assess the level of diffuse foregrounds, in particular synchrotron radiation and dust emission, as additional sources of systematic errors. Contamination from residual point sources is negligible. An estimate using the point-source component of the Planck Sky Model (PSM; Delabrouille et al. 2012, PSM v1.7.4) yields a limit of $C_{\ell}<1.4 \times 10^{-6} \mu \mathrm{K}^{2}$ over the entire $\ell$ range without masking any sources. ${ }^{31}$ An estimate based on a source-population model (Tucci \& Toffolatti 2012) relative to our nominal point-source mask results in an even lower level, $C_{\ell} \sim 5 \times 10^{-7} \mu \mathrm{K}^{2}$. Both are well below our statistical uncertainty.

Considering synchrotron radiation, we note that the $43 \mathrm{GHz}$ QUIET observations have already resulted in strong constraints on any synchrotron component in each of the QUIET CMB fields (QUIET 2011). Except for the single case of the $E E$ spectrum at $\ell \leqslant 75$ measured in CMB-1, no evidence of any contamination was found. These results allow us to constrain any contribution from synchrotron emission at $95 \mathrm{GHz}$ by extrapolation. Adopting a spectral index of $\beta_{\mathrm{s}}=-2.7$ (Dunkley et al. 2009), we estimate the $E E(B B)$ excess power to be $0.011 \pm 0.003 \mu \mathrm{K}^{2}\left(0.001 \pm 0.002 \mu \mathrm{K}^{2}\right)$ for the first bin of the CMB-1 spectrum, which is negligible compared to statistical errors.

In order to constrain contamination from dust emission, we adopt the thermal-dust component of the PSM as a template; the PSM predicts that other sources of contamination are subdominant at $95 \mathrm{GHz}$ in the QUIET fields. We estimate the dust power contribution in our fields by evaluating both the PSM power spectrum and the PSM-QUIET cross-spectrum using the PCL pipeline. The possible contamination is only relevant in the first bin $(25 \leqslant \ell \leqslant 75)$ of the field CMB-1. In this bin, the PSM power amplitude is $0.087 \mu \mathrm{K}^{2}\left(0.070 \mu \mathrm{K}^{2}\right)$ for the $E E(B B)$ spectrum, while the corresponding crosspower is $0.060 \pm 0.035 \mu \mathrm{K}^{2}\left(0.016 \pm 0.027 \mu \mathrm{K}^{2}\right)$. Taking into account the relative weights of the individual fields, we therefore estimate that the dust-emission contribution to the first $E E$ bin in the final co-added spectrum (Table 3 ) is $<0.04 \mu \mathrm{K}^{2}$, more than a factor two smaller than the statistical uncertainty. All other spectra and multipole ranges have negligible contributions. Fitting the PSM model as a template to CMB-1 in the map domain using the ML pipeline, we find a best-fit amplitude of $A=0.62 \pm 0.21$. This corresponds to a $3 \sigma$ correlation with the thermal-dust PSM component, which at the same time agrees with the PSM prediction $(A=1)$ at $1.8 \sigma$. Consistent results are obtained by taking the ratio of the cross-power to the PSM power including the full multipole range, with an amplitude of $A=0.66 \pm 0.25$. The three other fields all have best-fit amplitudes consistent with zero. We note as a caveat that the

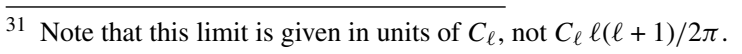

uncertainty in the PSM itself is not taken into account in this analysis, and the results depend critically on this model as the detected foreground levels are well below the statistical errors of the measured power spectra themselves.

\section{CONCLUSIONS}

We have presented the CMB polarization power spectra from the $95 \mathrm{GHz}$ QUIET observations. The $E E$ spectrum has been measured between $\ell=25$ and 975, and the first three acoustic peaks were seen with high signal-to-noise ratio, consistent with $\Lambda C D M$ predictions. The $B B$ spectrum was found to be consistent with zero, with a $95 \%$ C.L. upper limit on the tensorto-scalar ratio of $r<2.7$ (PCL) or 2.8 (ML), depending on pipeline. In Figure 6, we provide an up-to-date overview of the current state of the CMB polarization field, comparing the results from various experiments. ${ }^{32}$ In one of the fields, we found a correlation with the dust component of the PSM. The excess power due to this component was still small compared to the statistical errors of the power spectra. Finally, we have demonstrated the lowest level of instrumental systematic errors to date. We conclude by noting that part of the role of this experiment was to serve as a pathfinder to demonstrate that MMIC arrays were capable of reaching $r \lesssim 0.01$; this has been successfully achieved.

Support for the QUIET instrument and operation comes through the NSF cooperative agreement AST-0506648. Support was also provided by NSF awards PHY-0355328, AST-0448909, PHY-0551142, PHY-0855887, and AST1010016; KAKENHI 20244041, 20740158, and 21111002; PRODEX C90284; a KIPAC Enterprise grant; and by the Strategic Alliance for the Implementation of New Technologies (SAINT).

Some work was performed on the Joint Fermilab-KICP Supercomputing Cluster, supported by grants from Fermilab, the Kavli Institute for Cosmological Physics, and the University of Chicago. Some work was performed on the Titan Cluster, owned and maintained by the University of Oslo and NOTUR (the Norwegian High Performance Computing Consortium), and on the Central Computing System, owned and operated by the Computing Research Center at KEK. This research used resources of the National Energy Research Scientific Computing Center, which is supported by the Office of Science of the U.S. Department of Energy under Contract No. DE-AC02-05CH11231. Portions of this work were performed at the Jet Propulsion Laboratory (JPL) and California Institute of Technology, operating under a contract with the National Aeronautics and Space Administration. The $Q$-band modules were developed using funding from the JPL R\&TD program. We acknowledge the Northrop Grumman Corporation for collaboration in the development and fabrication of HEMT-based cryogenic temperature-compatible MMICs. We acknowledge the use of the Planck Sky Model, developed by the Component Separation Working Group (WG2) of the Planck Collaboration. Some of the results in this paper have been derived using the HEALPix (Gorski et al. 2005) software and analysis package.

C.D. acknowledges an STFC Advanced Fellowship and an ERC IRG grant under FP7. R.B. acknowledges support

\footnotetext{
32 For the $E E$ spectrum of QUIET, we show the mean of the spectrum from the two pipelines (after scaling to $q=1$ ) as a succinct visualization. For $B B$, the results from the two individual pipelines are indicated by the vertical extent of the QUIET-W points.
} 
from CONICYT project Basal PFB-06 and ALMA-Conicyt 31070015. A.D.M. acknowledges a Sloan foundation fellowship. H.K.E. acknowledges an ERC Starting Grant under FP7.

PWV measurements were provided by the Atacama Pathfinder Experiment (APEX). We thank CONICYT for granting permission to operate within the Chajnantor Scientific Preserve in Chile, and ALMA for providing site infrastructure support. Field operations were based at the Don Esteban facility run by Astro-Norte. We are particularly indebted to the engineers and technician who maintained and operated the telescope: José Cortés, Cristobal Jara, Freddy Muñoz, and Carlos Verdugo.

In addition, we acknowledge the following people for their assistance in the instrument design, construction, commissioning, operation, and in data analysis: Augusto Gutierrez Aitken, Colin Baines, Phil Bannister, Hannah Barker, Matthew R. Becker, Alex Blein, Mircea Bogdan, Anushya Chandra, Sea Moon Cho, Joelle Cooperrider, Mike Crofts, Emma Curry, Maire Daly, Richard Davis, Fritz Dejongh, Joy Didier, Greg Dooley, Hans Eide, Pedro Ferreira, Jonathon Goh, Will Grainger, Peter Hamlington, Takeo Higuchi, Seth Hillbrand, Christian Holler, Ben Hooberman, Kathryn D. Huff, William Imbriale, Oliver King, Eiichiro Komatsu, Jostein Kristiansen, Richard Lai, David Leibovitch, Erik Leitch, Kelly Lepo, Siqi Li, Martha Malin, Mark McCulloch, Steve Meyer, Oliver Montes, David Moore, Ian O’Dwyer, Gustavo Orellana, Stephen Osborne, Heather Owen, Stephen Padin, Felipe Pedreros, Ashley Perko, Alan Robinson, Jacklyn Sanders, Dale Sanford, Yunior Savon, Mary Soria, Alex Sugarbaker, David Sutton, Matias Vidal, Liza Volkova, Edward Wollack, Stephanie Xenos, Octavio Zapata, Mark Zaskowski, and Joseph Zuntz.

\section{REFERENCES}

Anderson, L., Aubourg, E., Bailey, S., et al. 2012, arXiv:1203.6594v1 Arnold, K., Ade, P. A. R., Anthony, A. E., et al. 2010, Proc. SPIE, $7741,77411 \mathrm{E}$

Aumont, J., Conversi, L., Thum, C., et al. 2010, A\&A, 514, A70

Benford, D. J., Chuss, D. T., Hilton, G. C., et al. 2010, Proc. SPIE, 7741, 77411Q Bischoff, C. A. 2010, PhD thesis, Univ. Chicago

Bischoff, C., Hyatt, L., McMahon, J. J., et al. 2008, ApJ, 684, 771

Bond, J. R., Jaffe, A. H., \& Knox, L. 1998, Phys. Rev. D, 57, 2117

Boyle, L. A., Steinhardt, P. J., \& Turok, N. 2006, Phys. Rev. Lett., 96, 111301

Brizius, A. R. 2011, PhD thesis, Univ. Chicago

Brown, M. L., Ade, P., Bock, J., et al. 2009, ApJ, 705, 978

Buder, I. D. 2012, PhD thesis, Univ. Chicago

Chiang, H. C., Ade, P. A. R., Barkats, D., et al. 2010, ApJ, 711, 1123

Chinone, Y. 2011, PhD thesis, Tohoku Univ.

Cleary, K. A. 2010, Proc. SPIE, 7741, 77412H

Cowan, G., Cranmer, K., Gross, E., \& Vitells, O. 2011, Eur. Phys. J. C, 71, 1554
Crill, B. P., Ade, P. A. R., Battistelli, E. S., et al. 2008, Proc. SPIE, 7010, 70102P Delabrouille, J., Betoule, M., Melin, J.-B., et al. 2012, arXiv:1207.3675

Dumoulin, R. N. 2011, PhD thesis, Columbia Univ.

Dunkley, J., Hlozek, R., Sievers, J., et al. 2011, ApJ, 739, 52

Dunkley, J., Spergel, D. N., Komatsu, E., et al. 2009, ApJ, 701, 1804

Eimer, J., Bennett, C., Chuss, D., et al. 2012, Proc. SPIE, 8452, 845220

Essinger-Hileman, T., Appel, J. W., Beal, J. A., et al. 2009, in AIP Conf. Ser. 1185, The Thirteenth International Workshop on Low Temperature Detectors-LTD13, ed. B. Young, B. Cabrera, \& A. Miller (Melville, NY: AIP), 494

Gorski, K. M., Hivon, E., Banday, A. J., et al. 2005, ApJ, 622, 759

Hansen, F. K., \& Gorski, K. M. 2003, MNRAS, 343, 559

Hicken, M., Wood-Vasey, W. M., Blondin, S., et al. 2009, ApJ, 700, 1097

Hivon, E., Gorski, K. M., Netterfield, C. B., et al. 2002, ApJ, 567, 2

Kamionkowski, M., Kosowsky, A., \& Stebbins, A. 1997, Phys. Rev. Lett., 78,2058

Keisler, R., Reichardt, C. L., Aird, K. A., et al. 2011, ApJ, 743, 28

Kessler, R., Becker, A. C., Cinabro, D., et al. 2009, ApJS, 185, 32

Klein, J. R., \& Roodman, A. 2005, Ann. Rev. Nucl. Part. Sci., 55, 141

Komatsu, E., Smith, K. M., Dunkley, J., et al. 2011, ApJS, 192, 18

Kusaka, A. 2010, Proc. the 45th Rencontre de Moriond, ed. E. Augé, J. Dumarchez, \& J. Trân Jhanh Vân (Hà Nội: Thế Gió'i), 7

Larson, D., Dunkley, J., Hinshaw, G., et al. 2011, ApJS, 192, 16

Leitch, E. M., Kovac, J. M., Halverson, N. W., et al. 2005, ApJ, 624, 10

Liddle, A. R., \& Lyth, D. H. 2000, Cosmological Inflation and Large-Scale Structure (Cambridge: Cambridge Univ. Press)

McMahon, J. J., Aird, K. A., Benson, B. A., et al. 2009, in AIP Conf. Ser. 1185 , The Thirteenth International Workshop on Low Temperature DetectorsLTD13, ed. B. Young, B. Cabrera, \& A. Miller (Melville, NY: AIP), 511

Monsalve, R. A. 2010, Proc. SPIE, 7741, 77412M

Monsalve, R. 2012, PhD thesis, Univ. Miami

Montroy, T. E., Ade, P. A. R., Bock, J. J., et al. 2006, ApJ, 647, 813

Næss, S. K. 2012, PhD thesis, Univ. Oslo

Newburgh, L. 2010, PhD thesis, Columbia Univ.

Newburgh, L. 2012, in Twelfth Marcel Grossmann Meeting on General Relativity, ed. T. Damour, R. T. Jantzen, \& Remo Ruffini (Singapore: World Scientific), 2145

Niemack, M. D., Ade, P. A. R., Aguirre, J., et al. 2010, Proc. SPIE, 7741, 77411S

O'Brient, R., Ade, P. A. R., Ahmed, Z., et al. 2012, Proc. SPIE, 8452, 84521G

Ogburn, R. W., IV, Ade, P. A. R., Aikin, R. W., et al. 2010, Proc. SPIE, $7741,77411 \mathrm{G}$

Oxley, P., Ade, P. A., Baccigalupi, C., et al. 2004, Proc. SPIE, 5543, 320

Padin, S., Shepherd, M. C., Cartwright, J. K., et al. 2002, PASP, 114, 83

Planck Collaboration, Ade, P. A. R., Aghanim, N., et al. 2011, A\&A, 536, A1 QUIET Collaboration. 2012, ApJ, submitted

QUIET Collaboration, Bischoff, C., Brizius, A., et al. 2011, ApJ, 741, 111

Reeves, R. 2012, J. Low Temp. Phys., 167, 929

Rozo, E., Wechsler, R. H., Rykoff, E. S., et al. 2010, ApJ, 708, 645

Seljak, U., \& Zaldarriaga, M. 1997, Phys. Rev. Lett., 78, 2054

Sheehy, C. D., Ade, P. A. R., Aikin, R. W., et al. 2010, Proc. SPIE, 7741, 77411R

Sievers, J. L., Achermann, C., Bond, J. R., et al. 2007, ApJ, 660, 976

Tajima, O., Nguyen, H., Bischoff, C., et al. 2012, J. Low Temp. Phys., 167,936

Tucci, M., \& Toffolatti, L. 2012, Adv. Astron., 624987

Wehus, I. K. 2012, in Proc. 23rd Rencontres de Blois: Particle Physics and Cosmology

Weiland, J. L., Odegard, N., Hill, R. S., et al. 2011, ApJS, 192, 19

Wu, J. H. P., Zuntz, J., Abroe, M. E., et al. 2007, ApJ, 665, 55 